\title{
Using High-k VPP Modes in Grating-Coupled Graphene-Based Hyperbolic Metamaterial for Tunable Sensor Design
}

This paper was downloaded from TechRxiv (https://www.techrxiv.org).

\section{LICENSE}

CC BY 4.0

SUBMISSION DATE / POSTED DATE

$21-01-2021 / 28-01-2021$

\section{CITATION}

Islam, Md Zahurul; Hoque, A.K.M. Hasibul; Sakib, Mashnoon Alam; Tsui, Ying Y. (2021): Using High-k VPP Modes in Grating-Coupled Graphene-Based Hyperbolic Metamaterial for Tunable Sensor Design. TechRxiv. Preprint. https://doi.org/10.36227/techrxiv.13619318.v1

$\mathrm{DOI}$ 


\title{
Using High- $k$ VPP Modes in Grating-Coupled Graphene-Based Hyperbolic Metamaterial for Tunable Sensor Design
}

\author{
A.K.M. Hasibul Hoque, Md Zahurul Islam, Mashnoon Alam Sakib, and Ying Tsui
}

\begin{abstract}
Volume plasmon polariton (VPP), a high-k mode that arises due to the coupling between two even modes of adjacent layers of an hyperbolic metamaterial (HMM) configuration, is very difficult to be excited by using prism coupling technique due to huge wave-vector mismatch. In this work, we present a graphenebased HMM stucture integrated with metal grating to facilitate excitation of VPP modes. A graphene HMM is composed of multilayer graphene super-lattice similar to metal-dielectric super-lattice structure. We report the analytical formulation of the dispersion relation and numerical results of the characteristics of the excited VPP modes for the proposed structure in the Terahertz region of the spectrum. The best achieved imaging resolution of our proposed structure is $15 \mathrm{~nm}$ when used as an infra-red imaging platform. As a sensing platform, a maximum sensitivity of 11,050 $\mathrm{nm} / \mathrm{RIU}$ is achieved for this configuration. The tunability of the resonance wavelength with respect to the structural parameters of the device is also studied and confirmed. Such promising findings are expected to make the proposed structure with integrated excitation coupler a potential candidate for tunable sensor design for different nanophotonic applications, including imaging, and, biomedical and chemical sensing applications.
\end{abstract}

Index Terms-Graphene-based Hyperbolic Metamaterial, Volume Plasmon Polariton, Plasmonic Sensor, Tunable Sensor Design

\section{INTRODUCTION}

$\mathbf{G}$ RAPHENE, a two-dimensional allotrope of carbon arranged in a honeycomb lattice [1], has attracted a lot of attention in the area of photonic research due to its extraordinary tunable electronic and optical properties. Electromagnetic waves, especially in the Terahertz region, can strongly interact with graphene electrons leading to the charged-oscillation of the carrier and creation of graphene plasmons [2,3]. The characteristics of graphene plasmons are different from its noblemetal counterpart due to its strong confinement [4,5], low loss [4], tunability [5,6] etc. For these exceptional properties of graphene plasmons, graphene has been studied extensively for its application in many areas, e.g., photo detection [7], optical modulation [8], sensing [9,10] and imaging [11] etc. Moreover, graphene based super lattices or metamaterials also show some exotic properties [12-14], which may expedite the commercialization of graphene based devices.

A.K.M. Hasibul Hoque and Md Zahurul Islam are with Department of Electrical and Electronic Engineering, Bangladesh University of Engineering and Technology, Dhaka - 1205, Bangladesh.

Mashnoon Alam Sakib is with Department of Electrical and Computer Engineering, University of Southern California, Los Angeles, USA

Ying Tsui is with Department of Electrical and Computer Engineering, University of Alberta, Edmonton, AB, Canada lam

Correspondence: mdzahurulislam@eee.buet.ac.bd, Md Zahurul Is-
Metamaterials are artificial sub-wavelength structures comprising of multiple materials, which can manifest unusual electromagnetic properties, such as negative refraction [15], superlensing [16] and electromagnetically-induced transparency (EIT) $[17,37]$. Numerous metamaterial configurations have been proposed particularly for sensing [18-21] applications. A special class of metamaterials is called hyperbolic metamaterials (HMMs) due to their hyperbolic dispersion relations. This specific type of dispersion arises from extreme anisotropy of the HMMs, one or two components of their permittivity/ permeability tensors being negative [22]. Several structures have been proposed to realize HMM, e.g., metaldielectric multilayer structure[23], nanowire arrays [23], hyperlens [23] and multilayer fishnet [23]. Different material combinations render HMM characteristics at different regions of the electromagnetic spectrum. HMMs can also be realized using graphene instead of regular metal/dielectric. A graphenedielectric multilayer structure can show hyperbolic dispersion property in the Terahertz region. This type of structures has been investigated both theoretically $[24,25]$ and experimentally [26]. Conventional HMMs have found some applications, for example sub-wavelength imaging [27], extreme sensing [28] and wave-guiding [29,30]. A metal-dielectric HMM can support high-k Volume plasmon polariton (VPP) or Bulk plasmon polariton (BPP) due to its diverging optical density of states. These VPP modes arise due to the coupling between 
Short-range (even) surface plasmon polaritons (SR-SPPs) in the individual metal-dielectric interface for metal-dielectric multilayer hyperbolic system [31]. It has been experimentally demonstrated that using a sub-wavelength diffraction grating, these VPP modes can be excited in regular metal-dielectric HMMs.

From theoretical perspective, graphene HMMs should also support VPP modes. Excitation of VPP modes in graphene HMMs is comparatively difficult due to a large wave-vector mismatch between VPP modes and free space wave. Using Kretschmann configuration demands a material with very high refractive index which is not physically possible yet. In this work, we propose a grating integrated graphene HMM device for the excitation of VPP modes in the Terahertz regime. A detailed theoretical and numerical study of thus excited VPP modes has been followed. Moreover, applicability of the proposed device for sensing and imaging purposes has been evaluated. For a silver (Ag) grating period of $1200 \mathrm{~nm}$, with graphene $\mathrm{Al}_{2} \mathrm{O}_{3} \mathrm{HMM}$ an imaging resolution of $15 \mathrm{~nm}$ has been achieved. As a sensor, a maximum sensitivity of $11,050 \mathrm{~nm} / \mathrm{RIU}$ has been observed for VPP modes around $3.57 \mathrm{THz}$. These metallic gratings induce a 'Fano resonance' in the reflectance and transmittance spectra. This is mainly due to the interaction between heavy-loss metallic plasmon modes and low-loss graphene plasmon modes.

\section{A. Proposed Grating Coupled Graphene HMM (GC-GHMM)}

The structure is illustrated in Fig. 1. Graphene HMM is composed of alternating layers of graphene-dielectric stack. Distance between any two consecutive graphene layers or width of dielectric layer is ' $\mathrm{d}$ ' $\mathrm{nm}$. A metallic grating is placed over the graphene HMM with a period of ' $a$ ' $\mathrm{nm}$, width ' $\mathrm{w}$ ' $\mathrm{nm}$ and height ' $h$ ' $\mathrm{nm}$. Light is incident from the top of grating (along negative $\mathrm{z}$ axis) when the device is simulated using Finite Difference Time Domain (FDTD) simulation technique. Grating coupling technique has used to fulfill the required phase-matching condition. A metal grating with a period of ' $a$ ' can overcome the mismatch between the in-plane wavevector of plasmon modes, $\beta$ and incident wave, $k_{0}$ [33].

$$
\beta=k_{0} \sin \theta \pm m \frac{2 \pi}{a} .
$$

Here $\theta$ indicates angle of incident, and ' $\mathrm{m}$ ' is a positive integer $(1,2,3 \ldots)$.

\section{Theoretical Analysis}

Strong interaction between photons and $\pi$-electrons of graphene creates charged oscillations known as graphene plasmons. Dispersion relation of graphene plasmons can be determined for TE and TM polarization as,

$$
\begin{gathered}
\text { TM mode: } \frac{i \sigma k}{\omega \epsilon_{0} \epsilon}+2=0 \\
\text { TE mode: } \frac{i \sigma \omega \mu_{0}}{k}+2=0
\end{gathered}
$$

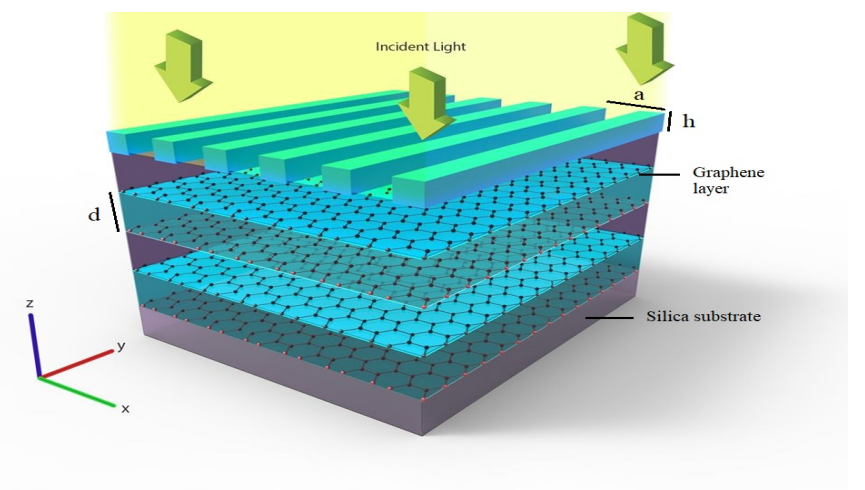

Fig. 1: Proposed Grating Coupled Graphene Hyperbolic Metamaterial. A metallic grating with period ' $a$ ' and height ' $h$ ' is at the top of graphene HMM. Graphene HMM is composed of 4 alternative graphene dielectric layers. Whole device is based on Silica substrate. Thickness of each dielectric layer is ' $d$ ' nm.

In-plane wave-vector of graphene plasmons can be calculated as $\beta=\sqrt{\epsilon k_{0}^{2}-k^{2}}, k_{0}$ is free space wave-vector. We consider a single layer graphene is surrounded by a dielectric with permittivity $\epsilon_{0} \epsilon$ and permeability $\mu_{0}$. Graphene conductivity can be calculated using expression in [34]. It is evident from equation that TM graphene plasmon mode has larger wave-vector (more confined) than its TE counterpart. This heavy confinement makes TM plsmon suitable for plasmonic application. As a result, for further analysis of VPP plasmons, TM plasmon is considered only. Coupling between these two plasmonic modes in graphene causes them to split into two modes, odd plasmon mode (Long-range surface plasmon polaritons (LR-SPPs) and even mode (Short-range surface plasmon polaritons (SR-SPPs). LR-SPPs have a lower wavevector than its single layer mode and SR-SPPs has a larger wave-vector. It has been shown by Zhukovsky et. al [31] that in metal-die multilayer HMMs, this SR-SPP mode evolve to VPP mode, allowing the mode to propagate in transverse direction of the multilayered structure. The dispersion relation for these two modes (LR-SPP and SR-SPP) for graphene plasmon are:

$$
\begin{aligned}
& \text { Odd mode: } \tanh (k d)=\frac{\sigma k}{i \omega \epsilon_{0} \epsilon}-1 \\
& \text { Even mode: } \tanh (k d)=\frac{1}{\frac{\sigma k}{i \omega \epsilon_{0} \epsilon}-1}
\end{aligned}
$$

' $\mathrm{d}$ ' is the distance between two graphene layers. The equations of real and imaginary parts of $\beta\left(=\sqrt{\epsilon k_{0}^{2}-k^{2}}\right)$ for both of these modes indicate that the even modes have longer wavevectors than those of the odd modes.

Decreasing the distance between the graphene layers causes the mode splitting to increase since the mode splitting depends on 'd'. From Fig (2a), it is clear that any decrease in 'd' casues SR-SPP modes to shift to lower frequency (or increasing wavevector at a specific frequency). Furthermore, with increased coupling, increasing $\beta$ makes it physically possible to evolve into high-k VPP modes in graphene HMM. 
(a)

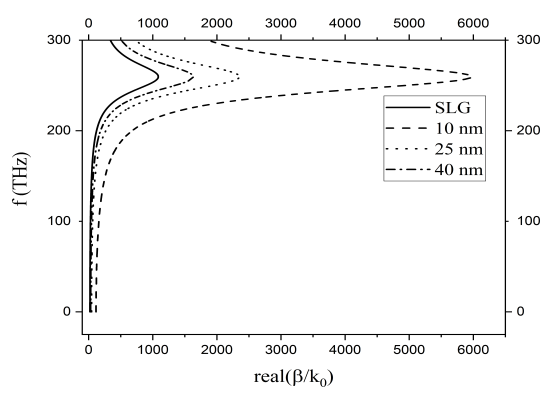

(b)

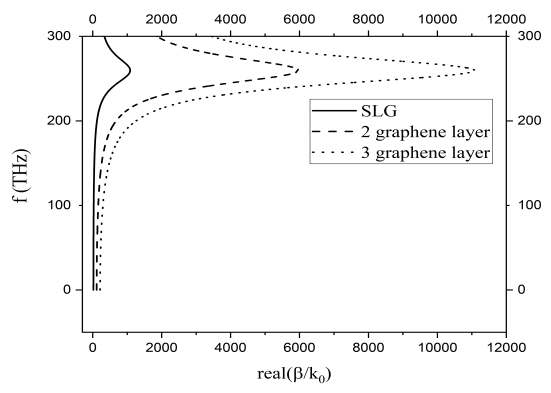

(c)

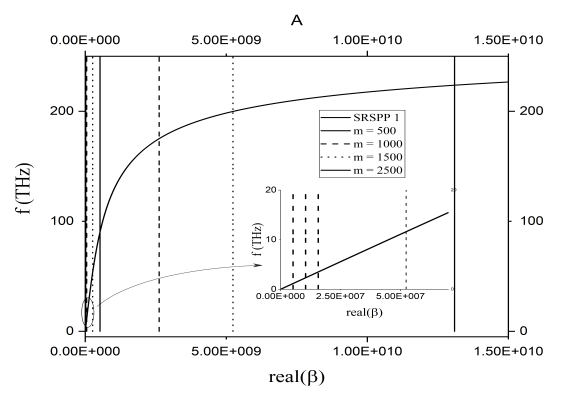

Fig. 2: Coupled graphene Plasmon (a) Real part of in-plane wave-vector for double layer of graphene with separation between graphene layers is 10, 25, $40 \mathrm{~nm}$. (b) Real part of LRSPP mode for different number of graphene layers (2, 3). (c) Using grating to excite plasmonic mode for three layers configuration. ' $\mathrm{m}$ ' is from equation (1). Inset shows the lower value of ' $\mathrm{m}$ ' $(\mathrm{m}=1,2,3,5)$.

Introducing three (3) alternate layers of graphene-dielectric further splits each mode. Dispersion relation for such a coupled graphene Plasmon mode among three alternate layers is:

$$
\exp (-2 k d)=\frac{\sigma k}{i \omega \epsilon_{0} \epsilon} \frac{\frac{\sigma k}{i \omega \epsilon_{0} \epsilon} \pm 4}{\left(\frac{\sigma k}{i \omega \epsilon_{0} \epsilon}-2\right)^{2}}
$$

Equation (6) is dispersion relation of four modes which can be written as,

$$
\begin{aligned}
\text { odd mode: } \tanh (k a) & =\frac{2-2 \frac{\sigma k}{i \omega \epsilon_{0} \epsilon_{r}}}{-\frac{\sigma^{2} k^{2}}{\omega^{2} \epsilon_{0}^{2} \epsilon_{r}^{2}}+2} \\
\text { even mode: } \tanh (k a) & =\frac{2}{\left(\frac{\sigma k}{i \omega \epsilon_{0} \epsilon_{r}}-2\right)^{2}}
\end{aligned}
$$

Equation (7) and (8) each contains two modes: even mode for three layers contains SR-SPP1 and SR-SPP2 and odd mode contains LR-SPP1 and LR-SPP2. One mode (SR-SPP1) shows further increase in wave-vector. This mode is specifically illustrated in Fig. 2(b). As the number of graphene layers is increased, one mode shows a gradual increase of wave-vector. This mode evolves to VPP mode for graphene HMM structure. All these modes have higher in-plane wave-vector than free space wave-vector (SR-SPP1 mode has $\beta / k_{0}=596 \mathrm{~nm}^{-1}$ at $2 \mathrm{THz}$ ). This huge mismatch can be overcome by coupling the incident wave through grating. A superimposed plot of SR-SPP1 dispersion with equation (1) with several ' $m$ ' values (using, $\theta=0$ ) and SR-SPP1 mode dispersion is shown in Fig 2(c). From the plot shown in inset, it is clear that $\mathrm{m}=3$ can excite SR-SPP1 mode at around $2 \mathrm{THz}$. At higher frequencies ( $>200 \mathrm{THz}$ ), excited modes in graphene is very closely spaced in the spectrum. Analytical calculation using more than three alternate graphene dielectric alternate layers is difficult. To understand the behavior of multilayered structure, FDTD method has been employed in this work. Graphene dielectric multilayer structure can support these high-k modes. Due to hyperbolic dispersion relationship of HMMs, these modes can propagate through the media in transverse direction. Transfer matrix for graphene can be written as,

$$
G=\left[\begin{array}{cc}
2-\frac{\sigma k}{\omega \epsilon_{0} \epsilon} & \frac{\sigma k}{\omega \epsilon_{0} \epsilon} \\
-\frac{\sigma k}{\omega \epsilon_{0} \epsilon} & 2+\frac{\sigma k}{\omega \epsilon_{0} \epsilon}
\end{array}\right]
$$

$\epsilon_{0} \epsilon$ is the permittivity of the dielectric media surrounding the graphene layer and $k$ is wave-vector in transverse direction. So the transfer matrix for a period of the HMM is $T=G D$, where $\mathrm{D}$ is the transfer matrix for the dielectric layer. We can determine the Bloch wave dispersion as, $\cos \mathbf{Q} d=\left(T_{11}+\right.$ $\left.T_{22}\right) / 2$, where $\mathbf{Q}$ is Bloch wave-vector. The dispersion relation of Bloch wave is:

$$
\cos (\mathbf{Q d})=\cos (\mathbf{k d})-\frac{\mathbf{i} \sigma \mathbf{k}}{\omega \epsilon_{\mathbf{0}} \epsilon} \sin (\mathbf{k d})
$$

By expanding the cosine function up to $k^{2} d^{2} / 2$ and the sine function up to $k d$, we get the dispersion relationship for the graphene-dielectric multilayer as,

$$
\frac{\mathbf{Q}^{2}}{\epsilon\left(\frac{1}{2}+\frac{i \sigma k}{\omega \epsilon_{0} \epsilon d}\right)}+\frac{\beta^{2}}{\epsilon}=k_{0}^{2}
$$

Hyperbolic dispersion appears when,

$$
\operatorname{Im}(\sigma)>\frac{\omega \epsilon_{0} \epsilon d}{2}
$$

For dielectric with $\epsilon=2.5$, hyperbolic region exists for frequency $<121 \mathrm{THz}(\lambda>2.5 \mu \mathrm{m})$. Increasing dielectric constant of the dielectric material causes the cut off frequency to decrease and vice versa. In the hyperbolic dispersion regime, a larger value of $\beta$ does not result in an imaginary value of $\mathbf{K}$. VPP mode can propagate in transverse direction inside HMM. In elliptical case, this transverse propagation is not possible, as higher $\beta$ value cause the wave to become evanescent in transverse direction, due to imaginary value of $\mathbf{K}$.

\section{Numerical AnAlysis}

\section{A. Methodology}

A 2D FDTD method has been employed to simulate the structure shown in Fig 1. Device is symmetric along y direction. Simulation has been performed in $x-z$ plane. Perfectly matched Layer (PML) has been employed along $\mathrm{z}$ direction. In the simulation, graphene was modeled as a $2 \mathrm{D}$ rectangle with zero thickness to reduce simulation time with sufficient accuracy of simulation results. A non-uniform meshing (finer mesh in the vicinity of interface) for the whole structure has 
(a)

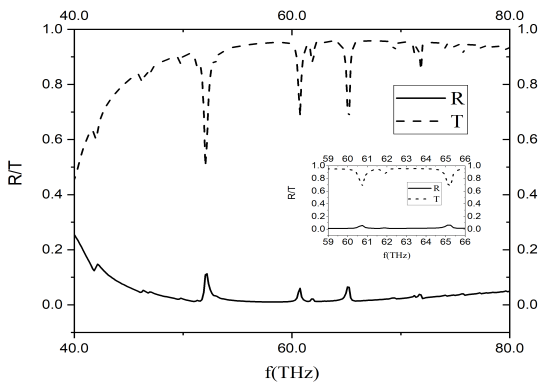

(b)

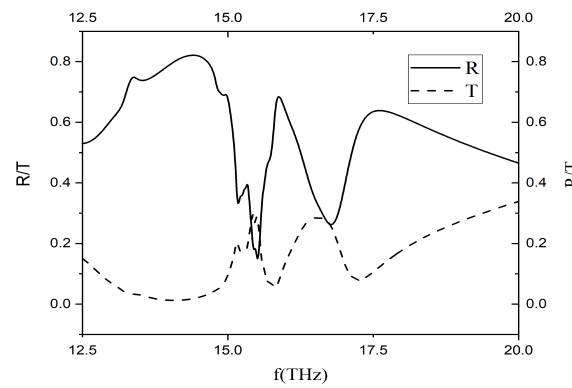

(c)

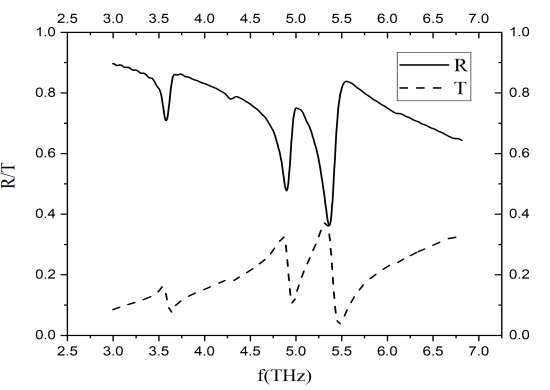

Fig. 3: Broadband reflectance and transmittance spectra (a) from 40 to $80 \mathrm{THz}$; (b) from 12.5 to $20 \mathrm{THz}$; (c) from 2.5 to 7 THz. Here, the GC-GHMM had 6 alternate layers of Graphene and $\mathrm{Al}_{2} \mathrm{O}_{3}$ with each $\mathrm{Al}_{2} \mathrm{O}_{3}$ layer has a thickness of 10 nm. Inset of (a) shows a closer look between 59 to $66 \mathrm{THz}$ with three modes.

been used to save computation time. An additional fine mesh of $2 \times 1 \mathrm{~nm}^{2}$ has been enforced in HMM region to get more accurate result. Conductivity of graphene has been computed from ref [31]. Unless otherwise specified, fermi energy and scattering rate of graphene is kept at $0.64 \mathrm{eV}$ and $0.196 \mathrm{meV}$, respectively. We only simulate the structure for TM polarized wave. Simulation has been performed for a broad range of frequency spanning from $2 \mathrm{THz}$ to $80 \mathrm{THz}$. In this region, the graphene HMM is at hyperbolic regime of dispersion. To reduce numerical artifact in the simulation, broadband simulation has been done in several steps. Light is incident on the GC-GHMM from the top (along $-\mathrm{z}$ axis according to Fig 1).

\section{B. Results}

Reflectance and transmission spectra of the proposed GCGHMM is shown in Fig3. Several peaks have been located in the transmittance spectrum corresponding to plasmonic VPP modes. Transmission has been measured along transverse direction of the HMM. High transmissivity in low frequency range indicates the propagating VPP mode. SR-SPPs cannot propagate along the transverse direction due its large imaginary value of transverse wave-vector. However, inside HMM, this is not the case as there is always a real value for transverse wave-vector for VPP mode. Fig 3 shows the case with graphene having chemical potential, $\mu=0.64 \mathrm{eV}$ and $\hbar \gamma=0.196 \mathrm{meV}$ using silver grating with period $800 \mathrm{~nm}$ and duty cycle of 0.5 . As we move towards higher frequencies, modes start to get closer to each other, which is clear from Fig 2(c). graphene- $\mathrm{Al}_{2} \mathrm{O}_{3} \mathrm{HMM}$ shows hyperbolic dispersion property for $f<70 \mathrm{THz}$. Using chemical potential, $\mu=$ $0.64 \mathrm{eV}$ makes graphene fully transparent at $\omega>2 \mu \approx 309$ THz. But a $97 \%$ transmission at $60 \mathrm{THz}$ marks the excitation of VPP modes inside HMM at that frequency.

Regarding resonance, asymmetry is found especially in lower frequency regime of transmittance spectrum (Fig 3(c)). This sort of asymmetry is due to the coupling between the metallic grating plasmons and graphene plasmon modes. Metal plasmon, a high ohmic loss plasmonic mode provides a broad spectrum while low-loss graphene plasmon gives a discrete spectrum, as discussed by Chen et.al [35]. From Fig2, resonance frequency can be tracked as $3.87,4.89,5.83,16.45$,
52, 60.7, 65.2 THz. There are several resonances beyond these points. The electric field (E-field) patterns at these resonances are analyzed using the same 2D FDTD technique. E-field pattern of VPP resonance is shown in Fig 4.

Propagation of the VPP modes along transverse direction is clear from Fig 4. Specifically, at low frequency regime, appearance of characteristic ' $\mathrm{V}$ ' angle is a signature of the excited VPP modes. This angle becomes more obvious in higher frequency regime (Fig 4(a), 4(b), 4(c)). In high frequency regime, plasmonic interference pattern appears due to interference between the two high-k modes of adjacent graphene layers. From Fig 2, it is obvious that high frequency plasmonic modes also have high in-plane wave-vectors. This extremely high wave-vector shows potential as an imaging platform. All these resonances experience different level of field enhancement and confinement as shown in table I. Field enhancement measures the maximum intensity of Efield that can be achieved for that particular resonant frequency. Moreover, field confinement measures the confinement ratio of graphene plasmons and resonant wavelength.Effective plasmonic wavelength is measured as the difference between two peak values in interference pattern. In case of 16.45 $\mathrm{THz}$, no plasmonic interference has been observed. At lower frequency, field confinement is increasing. However this will be a misleading result to use this device as imaging platform in that range. In order to achieve high resolution imaging platform, effective wavelength should be as low as possible which is only possible at high frequency for GC-GHMM. At low frequency, period for graphene plasmon increases, causes the resolution to increase. However, beyond a certain range, graphene plasmon loses its high-k characteristics (Fig 2(a)) and the wavelength gets larger. It is optimal to use GC-GHMM as imaging platform in 4-8 $\mu \mathrm{m}$ range.

The resonance mode of GC-GHMM is extremely tunable. Several parameters has been changed to investigate the tunable behavior of the GC-GHMM: dielectric material and its thickness, total number of alternate graphene-dielectric period/layers, chemical potential and scattering rate of graphene layer, period and duty cycle of grating. Effects of all these parameters have been analyzed using numerical 2D FDTD method. Reflectance spectrum from 2.5 to $7 \mathrm{THz}$ has been shown in Fig 5 for all these cases. 
(a)

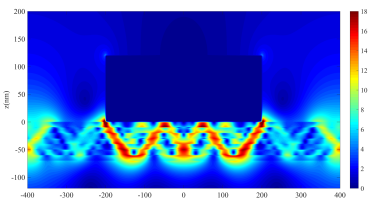

(e)

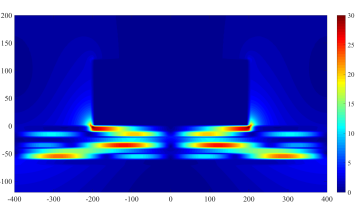

(b)

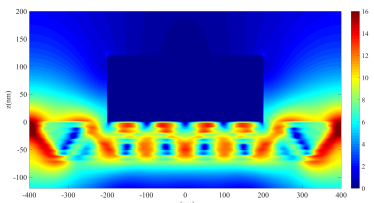

(f)

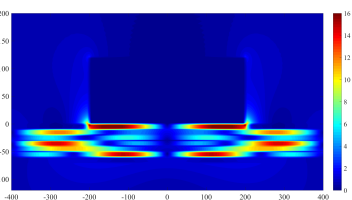

(c)

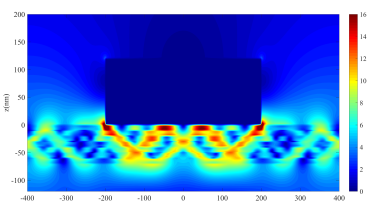

(g)

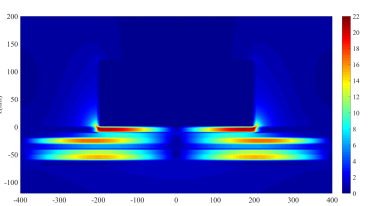

(d)

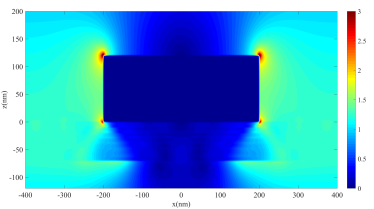

Fig. 4: Induced electric-field patterns at (a) $65.2 \mathrm{THz}$ (b) $60.7 \mathrm{THz}$ (c) $52 \mathrm{THz}$ (d) $16.45 \mathrm{THz}$ (e) $5.36 \mathrm{THz}$ (f) $4.89 \mathrm{THz}$ (g) 3.57 THz. Light is incident from the top.

(a)

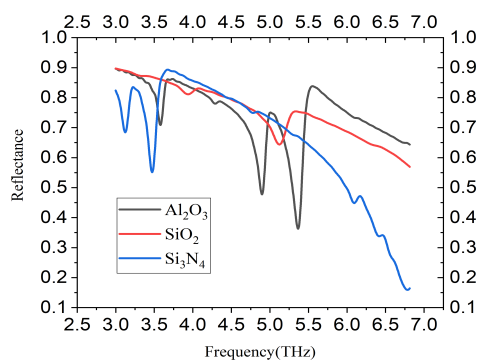

(d)

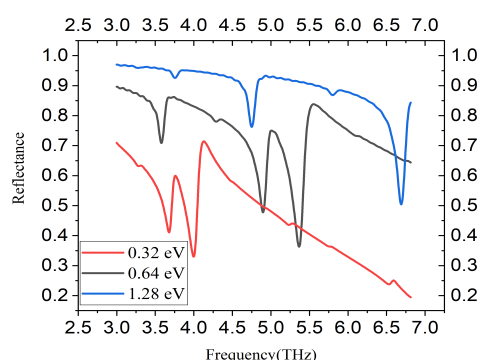

(b)

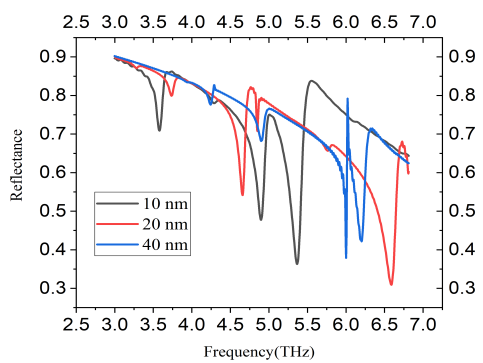

(e)

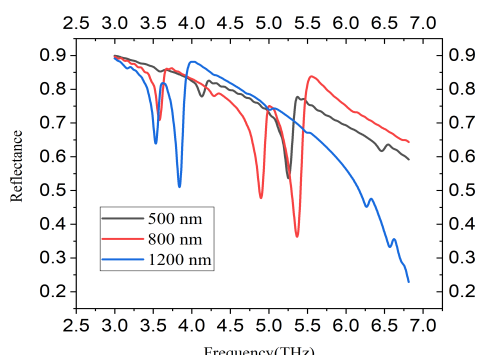

(c)

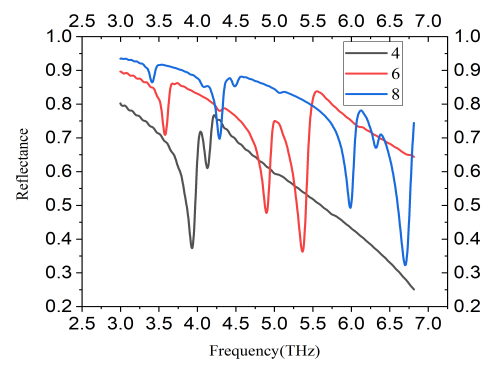

(f)

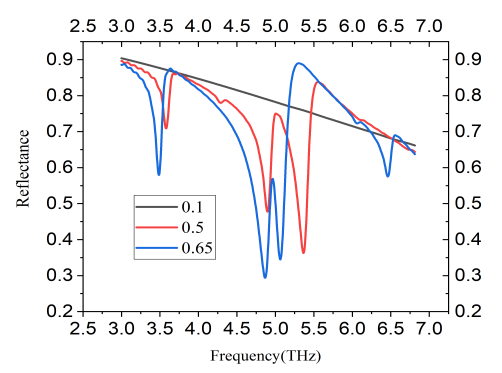

Fig. 5: Reflectance spectra for (a) different dielectrics, (b) different dielectric thickness, (c) different number of graphene die periods, (d) different chemical potential of Graphene, (e) different grating period, and (f) different grating duty cycle.

TABLE I: Plasmonic field confinement and enhancement at different frequencies.

\begin{tabular}{|l|l|l|l|}
\hline $\begin{array}{l}\text { Resonance } \\
\text { frequency } \\
(\mathrm{THz})\end{array}$ & $\begin{array}{l}\text { Field en- } \\
\text { hancement }\end{array}$ & $\begin{array}{l}\text { Effective wavelength of } \\
\text { Graphene Plasmon (nm) }\end{array}$ & $\begin{array}{l}\text { Field } \\
\text { confinement }\end{array}$ \\
\hline 65.2 & 18 & 100 & 46 \\
\hline 60.7 & 16 & 96 & 51 \\
\hline 52 & 16 & 104 & 62 \\
\hline 16.45 & 3 & - & - \\
\hline 5.36 & 30 & 385 & 145 \\
\hline 4.89 & 16 & 345 & 175 \\
\hline 3.57 & 22 & 380 & 221 \\
\hline
\end{tabular}

Increasing dielectric permittivity causes the HMM region to red shift (equation (12)). Same trend can be found from equation (2), (5) and (8). From Fig. 5(a), it is clear that same red shift has occurred in the numerical simulation using the dielectric material with increasing dielectric constant $\left(\mathrm{SiO}_{2}, \mathrm{Al}_{2} \mathrm{O}_{3}, \mathrm{Si}_{3} \mathrm{~N}_{4}\right.$ in that order). With increasing thickness of dielectric layer, coupling between graphene layer is weakening causing the graphene plasmon mode shift to higher frequency. Furthermore, increasing distance causes some high order plasmonic excitation to disappear that mode is not available (Fig 2(a)). But blue shift with increasing thickness is obvious from Fig 5(b). Increasing the number of graphenedie layers makes dispersion of the HMM material an ideal hyperbolic dispersion [24]. This idea has also been cleared in Fig. 2(b). Clear redshift from Fig 5(c) supports that statement. Changing chemical potential of graphene causes graphene optical conductivity to change. Increasing chemical potential causes the inter-band transition to occur at higher frequency, resulting an overall blueshift for the resonant mode. Effect of grating period is explained in equation (1). Changing period 
(a)

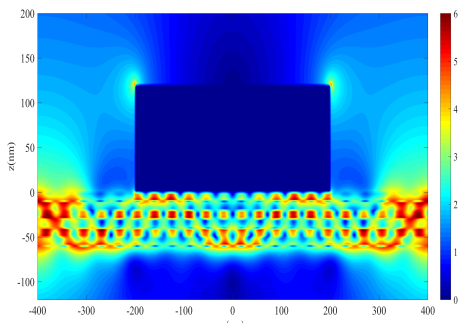

(d)

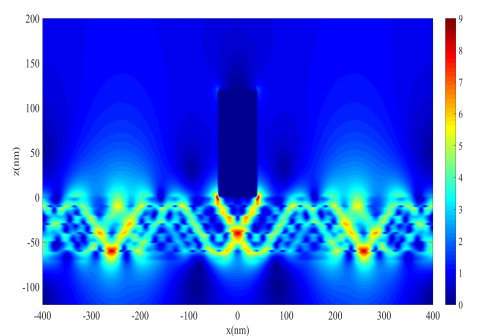

(b)

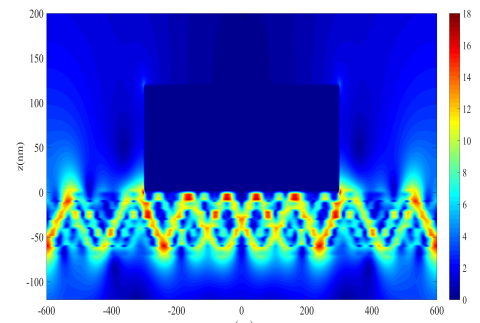

(e)

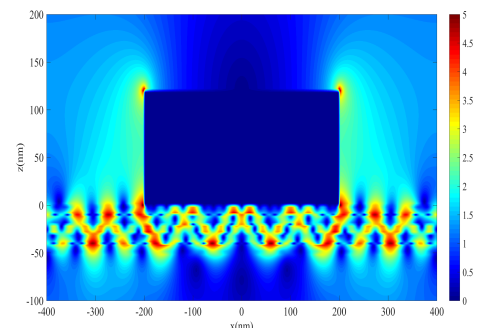

(c)

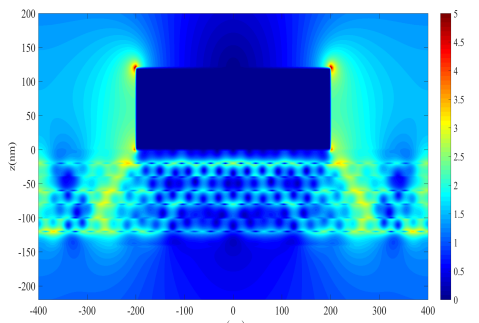

(f)

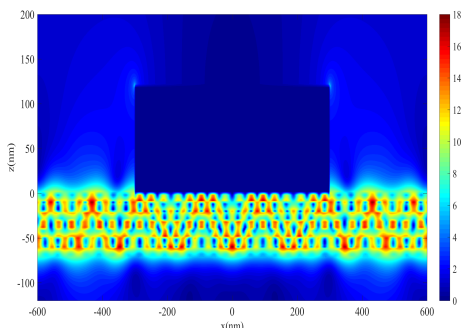

Fig. 6: Plasmonic interference pattern for (a) Graphene-10 nm $\mathrm{SiO}_{2} \mathrm{HMM}$ at $65.57 \mathrm{THz}$, (b) Graphene-10 nm $\mathrm{Al}_{2} \mathrm{O}_{3}$ with Ag grating of $1200 \mathrm{~nm}$ at $57.6 \mathrm{THz}$, (c) Graphene-20 nm $\mathrm{Al}_{2} \mathrm{O}_{3}$ at $60.5 \mathrm{THz}$, (d) Graphene-10 nm $\mathrm{Al}_{2} \mathrm{O}_{3}$ with 0.1 grating duty cycle at $64.5 \mathrm{THz}$, (e) four alternate layers of Graphene-10 nm $\mathrm{Al}_{2} \mathrm{O}_{3}$ at $64.6 \mathrm{THz}$, and (f) Graphene-10 nm $\mathrm{Al}_{2} \mathrm{O}_{3} \mathrm{with}$ Ag grating of $1200 \mathrm{~nm}$ at $65.13 \mathrm{THz}$.

can cause several new modes to appear as excitation with new wavevector is possible. Some of these is visible in Fig 5(e). Changing the duty cycle does not affect the position of resonant mode too much. But decreasing it to a low value can cause the perturbation of incident wave front insignificant to cause the incident wave to excite plasmonic mode in graphene at low frequency. This tunability of graphene VPP mode is a good criterion for GC-GHMM to be used as sensor. Slight change in dielectric constant can cause the VPP resonance mode to shift in frequency and can be exploited as a sensor.

\section{GC-GHMM as imaging Platform}

As hinted earlier, GC-GHMM can be used as imaging platform in wavelength range of 4-8 $\mu \mathrm{m}$. To find out the optimal condition of GC-GHMM all the parameters mentioned above has been tried and interference pattern along with field enhancement has been calculated. Several electric field profiles have been shown in fig. 6 .

For an $\mathrm{Ag}$ grating with a period of $800 \mathrm{~nm}$ and a duty cycle of 0.5, plasmonic interference pattern appears at a distance of $35 \mathrm{~nm}$ (Fig. 6 (a)) in for GHMM with $10 \mathrm{~nm} \mathrm{SiO}_{2}$ at incident wavelength of $4.57 \mu \mathrm{m}(65.57 \mathrm{THz})$ (Fig 6(a)). A six times enhancement of field intensity and 130-fold increase in the field confinement have been achieved which is way beyond the diffraction limit with imaging resolution of $18 \mathrm{~nm}$. With increasing dielectric thickness, overlapping between adjacent graphene plasmons diminishes and interference pattern eventually vanishes (Fig 6(c)). For an Ag grating with a period of $1200 \mathrm{~nm}$ and a duty cycle of 0.5 , plasmonic interference pattern appears at a distance of $30 \mathrm{~nm}(07$ (b)) for $4.6 \mu \mathrm{m}$ wavelength excitation, which results in 153-fold increase in the confinement of the incident wave (Fig 6(f)) and an imaging resolution of $15 \mathrm{~nm}$. All these result makes GC-GHMM a strong candidate for infrared imaging platform. Interference pattern actually change with grating property (period and duty cycle).

\section{GC-GHMM as sensing Platform}

We utilize the spectral change of resonance mode with refractive index to use GC-GHMM as a sensor. For proof of concept, a graphene- $\mathrm{Al}_{2} \mathrm{O}_{3} \mathrm{HMM}$ with 6 alternate periods of single-layer graphene-10 $\mathrm{nm} \mathrm{Al}_{2} \mathrm{O}_{3}$ has been studied for its performance as a sensing device where the third $\mathrm{Al}_{2} \mathrm{O}_{3}$ layer is used as the sensor platform, i.e this layer has been replaced with sensing layer. FDTD simulation has been performed for this structure for three different refractive indices of the sensing layer; $1.3,1.4$ and 1.5. Results of the simulation is shown in Table II. The VPP modes at low frequencies show a relatively higher sensitivity compared to the modes at higher frequencies. The spectral shift is also depicted in Fig. 8. As can be seen from Table II, a high sensitivity of $11050 \mathrm{~nm} / \mathrm{RIU}$ can be obtained from this sensor configuration.

\section{CONCLUSION}

In this report, we propose a graphene-based HMM structure with integrated metal grating for efficient coupling of the electromagnetic wave to the structure to facilitate efficient excitation of high- $k$ VPPs. The analytical expression of dispersion relation for the proposed structure is formulated and has found to be indicated the origins of these sort of modes in the structure. To validate the analytical results, numerical simulation has been performed and field confinement and enhancement of the VPP modes have been determined. 
(a)

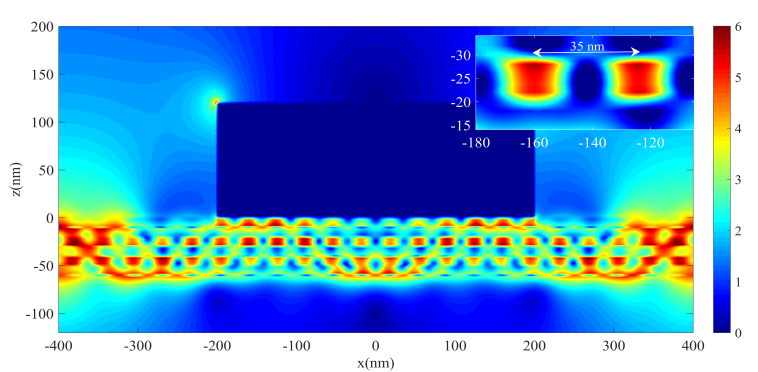

(b)

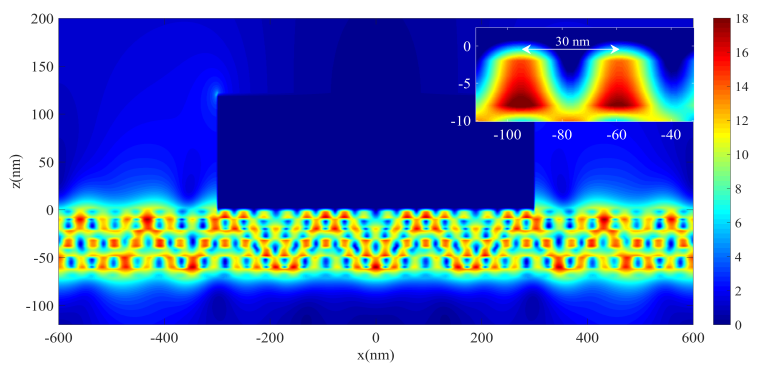

Fig. 7: Plasmonic interference pattern of GC-GHMM. (a) Using silver grating with period $800 \mathrm{~nm}$, duty cycle 0.5 on graphene-10 nm $\mathrm{SiO}_{2}$ HMM. (b) Using silver grating with period $1200 \mathrm{~nm}$, duty cycle 0.5 on graphene- $10 \mathrm{~nm} \mathrm{Al}_{2} \mathrm{O}_{3}$ HMM. Inset in both figures show two interference peaks. Distance between the two peaks is also shown.

(a)

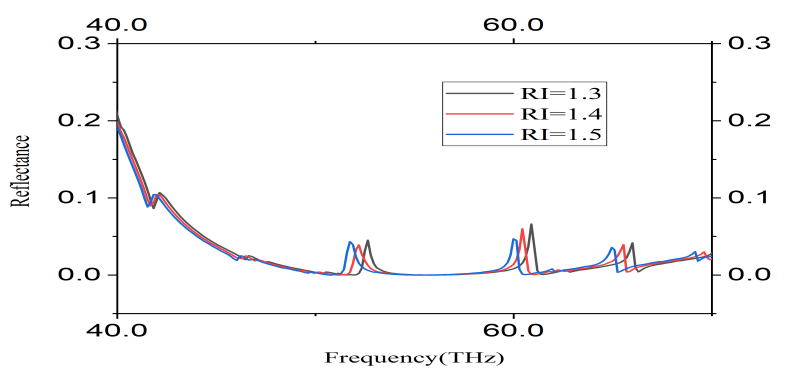

(b)

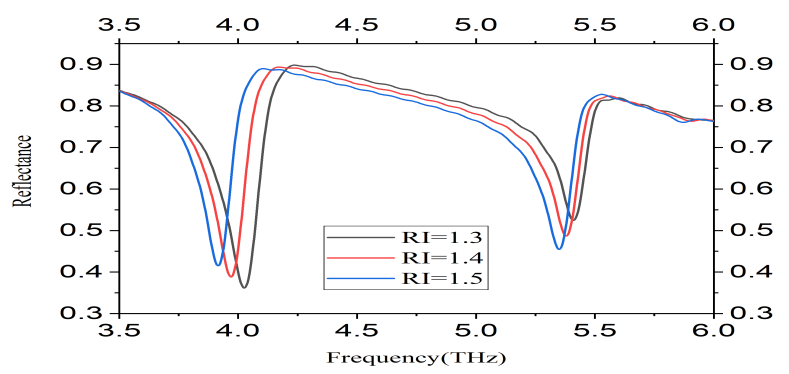

Fig. 8: Reflectance spectrum of GC-GHMM for three different refractive indices.

Plasmonic interference pattern is observed in the nano-cavity formed between adjacent graphene layers. This enhanced plasmonic interference phenomena of the proposed structure is further employed to be used as an imaging platform in
TABLE II: Sensitivity of different VPP modes.

\begin{tabular}{|l|l|l|}
\hline $\begin{array}{l}\text { Refractive } \\
\text { index (RI) } \\
\text { of the } \\
\text { sensing } \\
\text { layer }\end{array}$ & $\begin{array}{l}\text { Resonance } \\
\text { position } \\
(\mathrm{nm})\end{array}$ & $\begin{array}{l}\text { Sensitivity } \\
(\mathrm{nm} / \text { RIU) }\end{array}$ \\
\hline 1.3 & 4542 & - \\
\hline 1.4 & 4573 & 310 \\
\hline 1.5 & 4605 & 320 \\
\hline \hline 1.3 & 4922 & - \\
\hline 1.4 & 4959 & 370 \\
\hline 1.5 & 4984 & 250 \\
\hline \hline 1.3 & 5694 & - \\
\hline 1.4 & 5760 & 370 \\
\hline 1.5 & 5794 & 250 \\
\hline \hline 1.3 & 55419 & - \\
\hline 1.4 & 55714 & 2950 \\
\hline 1.5 & 56063 & 3490 \\
\hline \hline 1.3 & 74489 & - \\
\hline 1.4 & 75474 & 9850 \\
\hline 1.5 & 76579 & 11050 \\
\hline
\end{tabular}

the infra-red regime. Furthermore, it has been shown that the periodic pattern of the plasmonic interference and the spectral position of the VPP modes can be tuned in several ways and thus it provides additional degrees of freedom in designing appropriate resonance wavelength for the desired applications.

\section{RefEREnCES}

[1] Neto, A. C., Guinea, F., Peres, N. M., Novoselov, K. S. \& Geim, A. K "The electronic properties of graphene." Rev. modern physics, 81, 109 (2009).

[2] Grigorenko, A., Polini, M. \& Novoselov, K. "Graphene plasmonics." Nat. photonics, 6, 749 (2012).

[3] Koppens, F. H., Chang, D. E. \& Garcia de Abajo, F. J. "Graphene plasmonics: a platform for strong light-matter interactions." Nano letters 11,,3370-3377 (2011).

[4] Jablan, M., Buljan, H. \& Solja `ci 'c, "M. Plasmonics in graphene at infrared frequencies." Phys. review B, 80, 245435 (2009).

[5] Chen, J. et al. "Optical nano-imaging of gate-tunable graphene plasmons." Nature, 487, 77 (2012).

[6] Ju, L. et al. "Graphene plasmonics for tunable terahertz metamaterials." Nat. nanotechnology 6, 630 (2011).

[7] Mueller, T., Xia, F. \& Avouris, P. "Graphene photodetectors for highspeed optical communications." Nat. photonics 4, 297 (2010).

[8] "A graphene-based broadband optical modulator.” Liu, M. et al. Nature 474, 64 (2011).

[9] Zhao, Y. \& Zhu, Y. Graphene-based hybrid films for plasmonic sensing. Nanoscale 7, 14561-14576 (2015).

[10] Zeng, S. et al. "Graphene-gold metasurface architectures for ultrasensitive plasmonic biosensing." Adv. Mater. 27, 6163-6169 (2015).

[11] Yang, J., Wang, T., Chen, Z., Hu, B. \& Yu, W. "Super-resolution imaging at mid-infrared waveband in graphene-nanocavity formed on meta-surface." Sci. reports 6, 37898 (2016).

[12] Yan, $H$. et al. Tunable infrared plasmonic devices using graphene/insulator stacks. Nat. nanotechnology 7, 330 (2012).

[13] Vakil, A. \& Engheta, N. Transformation optics using graphene. Science 332, 1291-1294 (2011).

[14] Lee, S. H. et al. "Switching terahertz waves with gate-controlled active graphene metamaterials." Nat. materials 11, 936 (2012).

[15] Shalaev, V. M. "Optical negative-index metamaterials." Nat. photonics 1, 41 (2007).

[16] Kawata, S., Inouye, Y. \& Verma, P. "Plasmonics for near-field nanoimaging and superlensing." Nat. Photonics 3, 388 (2009).

[17] Luk'yanchuk, B. et al. The fano resonance in plasmonic nanostructures and metamaterials. Nat. materials 9, 707 (2010).

[18] Chen, T., Li, S. \& Sun, H. Metamaterials application in sensing. Sensors 12, 2742-2765 (2012).

[19] Yousuf, S. E. H., Sakib, M. A. \& Islam, M. Z. A high-performance plasmonic nanosensor based on an elliptical nanorod in an mim configuration. IEEE Sensors J. 18, 6145-6153 (2018). 
[20] Sakib, M. A., Yousuf, S. E. H., Gupta, S. D. \& Islam, M. Z. Proposition and numerical analysis of a plasmonic sensing structure of metallodielectric grating and silver nano-slabs in a metal-insulator-metal configuration. Plasmonics 13, 2205-2213 (2018).

[21] Al-mahmod, M. J., Hyder, R. \& Islam, M. Z. Numerical studies on a plasmonic temperature nanosensor based on a metalinsulator- metal ring resonator structure for optical integrated circuit applications. Photonics Nanostructures-Fundamentals Appl. 25, 52-57 (2017).

[22] Shekhar, P., Atkinson, J. \& Jacob, Z. Hyperbolic metamaterials: fundamentals and applications. Nano Convergence 1, 1-17 (2014).

[23] Poddubny, A., Iorsh, I., Belov, P. \& Kivshar, Y. Hyperbolic metamaterials. Nat. photonics 7, 948 (2013).

[24] Iorsh, I. V., Mukhin, I. S., Shadrivov, I. V., Belov, P. A. \& Kivshar, Y $\mathrm{S}$. Hyperbolic metamaterials based on multilayer graphene structures. Phys. Rev. B 87, 075416 (2013).

[25] Othman, M. A., Guclu, C. \& Capolino, F. Graphene-based tunable hyperbolic metamaterials and enhanced near-field absorption. Opt. express 21, 7614-7632 (2013).

[26] Chang, Y.-C. et al. Realization of mid-infrared graphene hyperbolic metamaterials. Nat. communications 7, 10568 (2016).

[27] Xiong, Y., Liu, Z. \& Zhang, X. A simple design of flat hyperlens for lithography and imaging with half-pitch resolution down to $20 \mathrm{~nm}$. Appl. Phys. Lett. 94, 203108 (2009)

[28] Sreekanth, K. V. et al. Extreme sensitivity biosensing platform based on hyperbolic metamaterials. Nat. materials 15, 621 (2016).

[29] Zhang, Z. \& Fan, Y. Propagation properties of a wave in a disordered multilayered system containing hyperbolic metamaterials. JOSA B 29 , 2995-2999 (2012).

[30] He, Y., He, S. \& Yang, X. Optical field enhancement in nanoscale slot waveguides of hyperbolic metamaterials. Opt. letters 37, 2907-2909 (2012).

[31] Zhukovsky, S. V., Kidwai, O. \& Sipe, J. Physical nature of volume plasmon polaritons in hyperbolic metamaterials. Opt. express 21 , 14982-14987 (2013).

[32] Avouris, P. \& Xia, F. Graphene applications in electronics and photonics. Mrs Bull. 37, 1225-1234 (2012).

[33] Maier, S. A. Plasmonics: fundamentals and applications (Springer Science \& Business Media, 2007)

[34] Hanson, G. W. Dyadic green's functions and guided surface waves for a surface conductivity model of graphene. J. Appl. Phys. 103, 064302 (2008).

[35] Chen, Z.-x. et al. Tunable fano resonance in hybrid graphene-metal gratings. Appl. Phys. Lett. 104, 161114 (2014).

[36] Ishii, S., Kildishev, A. V., Narimanov, E., Shalaev, V. M. \& Drachev, V. P. Sub-wavelength interference pattern from volume plasmon polaritons in a hyperbolic medium. Laser \& Photonics Rev. 7, 265-271 (2013).

[37] Baqir, M.A., Choudhury, P.K., Farmani, A., Younas, T., Arshad, J., Mir, A. and Karimi, S., 2019. Tunable plasmon induced transparency in graphene and hyperbolic metamaterial-based structure. IEEE Photonics Journal, 11(4), pp.1-10. 